\title{
VALIDACIÓN DEL MÓDULO WHOQOL-OLD EN PERSONAS ATENDIDAS EN ORGANIZACIONES PARA EL ADULTO MAYOR
}

\author{
Aurora Molina Napurí ${ }^{1}$ y Emma Cambillo Moyano ${ }^{2}$
}

\begin{abstract}
Resumen: WHOQOL-OLD es un instrumento diseñado por la OMS para medir la calidad de vida en adultos mayores. Objetivo: Validar el módulo WHOQOL-OLD para evaluar la calidad de vida de personas atendidas en organizaciones para el Adulto Mayor del distrito de Miraflores. Método: El estudio es de enfoque cuantitativo, no experimental, de corte transversal. El diseño muestral es probabilístico y tamaño de muestra de 325 personas adultas mayores seleccionadas aleatoriamente de los tres Centros de Atención al Adulto Mayor del distrito de Miraflores. Para validar el módulo WHOQOL-OLD, se hizo uso del modelo de ecuaciones estructurales en tres etapas detalladas en el documento. Resultados: Mediante el método de estimación por Mínimos Cuadrados de libre distribución, la Calidad de Vida aplicando el Módulo WHOQOL-OLD de la OMS, influye directa y positivamente sobre cinco de sus seis factores, con índices de ajuste satisfactorios ( $\mathrm{GFI}=0,94 ; \mathrm{SRMR}=0,10 ; \mathrm{CFI}=0,94$; $\mathrm{NFI}=0,90 ; \mathrm{PGFI}=0,70)$. Conclusiones: El Módulo WHOQOL-OLD de la OMS, no fue posible validarlo totalmente, siendo que la faceta "Actividades en el tiempo" no presentó el aporte necesario para su validación.
\end{abstract}

Palabras clave: Modelo de Ecuaciones Estructurales; Calidad de Vida.

\section{VALIDATION OF THE WHOQOL-OLD MODULE IN PEOPLE CARED FOR IN ORGANIZATIONS FOR THE ELDERLY ADULT}

\begin{abstract}
WHOQOL-OLD is an instrument designed by the WHO to measure quality of life in older adults. Objective: To validate the WHOQOL-OLD module to evaluate the quality of life of people cared for in organizations for the Elderly in the Miraflores district. Method: The study has a quantitative, non-experimental, cross-sectional approach. The sample design is probabilistic and a sample size of 325 elderly people randomly selected from the three Elderly Care Centers in the district of Miraflores. To validate the WHOQOL-OLD module, the structural equation model was used in three stages detailed in the document. Results: By means of the estimation method by Least Squares of free distribution, the Quality of Life applying the WHOQOL-OLD Module of the WHO, directly and positively influences five of its six factors, with satisfactory adjustment indices $(\mathrm{GFI}=0.94 ; \mathrm{SRMR}=0.10$; $\mathrm{CFI}=0.94 ; \mathrm{NFI}=0.90 ; \mathrm{PGFI}=0.70$ ). Conclusions: The WHOQOL-OLD Module of the WHO, it was not possible to validate it completely, since the facet.${ }^{\mathrm{A}} \mathrm{ctivities}$ in time" did not present the necessary contribution for its validation.
\end{abstract}

Keywords: Structural Equation Models; Quality of Life.

Recibido: 25/01/2021. Aceptado: 14/04/2021. Publicado online: 30/06/2021.

(C) Los autores. Este artículo es publicado por la Revista PESQUIMAT de la Facultad de Ciencias Matemáticas, Universidad Nacional Mayor de San Marcos. Este es un artículo de acceso abierto, distribuido bajo los términos de la licencia Creative Commons Atribucion-No Comercia-CompartirIgual 4.0 Internacional.(http://creativecommons.org/licenses/by-nc-sa/4.0/) que permite el uso no comercial, distribución y reproducción en cualquier medio, siempre que la obra original sea debidamente citada. Para uso comercial, por favor póngase en contacto con revistapesquimat.matematica@unmsm.edu.pe

${ }^{1}$ UNMSM, Facultad de Ciencias Matemáticas. e-mail: aurora.molina@unmsm.edu.pe

${ }^{2}$ UNMSM, Facultad de Ciencias Matemáticas, e-mail: ecambillom@unmsm.edu.pe 


\section{Introducción}

La Organización Mundial de la Salud (OMS) refiriéndose al envejecimiento, manifiesta:

Debido al incremento de la esperanza de vida y a la disminución de la tasa de fecundidad, la proporción de personas mayores de 60 años está aumentando más rápidamente que cualquier otro grupo de edad en casi todos los países. El envejecimiento de la población puede considerarse un éxito de las políticas de salud pública y el desarrollo socio económico, pero también constituye un reto para la sociedad, que debe adaptarse a ello para mejorar al máximo la salud y la capacidad funcional de las personas mayores, así como su participación social y su seguridad. [1, párr.1]

En el Perú, en la década de los años cincuenta, la población estaba compuesta básicamente por niños y niñas menores de 15 años de edad (42\%), presentándose una drástica disminución en el 2020 (25\%); mientras que, en este mismo periodo, el proceso de envejecimiento de la población adulta mayor fue en aumento (de 5,7\% a 12,7\%). Además, en Lima Metropolitana (2020) la proporción de hogares con algún miembro adulto mayor alcanzó el 46,5\%. [2, p. 1-2].

Cabe destacar que, la presente validación se basa en la muestra recogida por Molina [3] para el estudio "Relación entre el nivel de dependencia de funcionalidad de las actividades cotidianas y la calidad de vida de personas atendidas en organizaciones para el adulto mayor", conformado por personas de la tercera edad (60 años a más) sin problemas cognitivos, siendo su enfoque principal determinar la relación entre Calidad de Vida y la Dependencia de Funcionalidad. Estudios similares se basan en el Módulo WHOQOL-OLD y su relación entre diversas situaciones de salud como: "Calidad de vida y su relación con la desnutrición en el adulto mayor de la provincia de Ica" 4], "Calidad de vida en el adulto mayor con enfermedades crónicas en el grupo - Un día más" [5], "La actividad física como intervención para la mejora de la calidad de vida en el adulto mayor" [6], entre muchos otros que muestran diferencias tangibles en la Calidad de Vida según estudio, llevando a considerar posibles cambios estructurales en el instrumento.

Por otro lado, en el enfoque del envejecimiento, el Tercer Plan Nacional de Derechos Humanos [7. p. 59] elaboró un plan de Derechos que se incorporó a la población adulta mayor como grupo de especial protección, proponiendo acciones estratégicas que contribuyan a cumplir con las políticas públicas diseñadas por el Estado, y con ello, estudios como "Medición de la calidad de vida en adultos mayores institucionalizado de Lima" [8], el "Nivel de dependencia y la percepción de la calidad de vida de los adultos mayores del centro de atención residencial geriátrico San Vicente de Paúl" [9, el "Calidad de vida y estrategias de afrontamiento en adultos mayores de Lima Metropolitana" [10, entre otros; muestran cómo la aplicación del Módulo WHOQOLOLD arroja resultados que pueden ser considerados para obtener información sobre la Calidad de Vida del adulto mayor, siendo importante por ello, validar dicho instrumento.

\section{Materiales y Métodos}

\subsection{Materiales}

El instrumento de medición utilizado fue el Módulo WHOQOL-OLD elaborado por la OMS, quienes autorizaron su aplicación y análisis para la investigación en referencia.

La OMS en un estudio realizado por Power, Quinn y Schmidt en el 2006 [11, presenta el desarrollo de un módulo adicional para las medidas de la calidad de vida (QoL) de WHOQOL para su uso con adultos mayores, conocido como WHOQOL-OLD [12], el cual está distribuido en seis facetas con cuatro ítems cada uno, valorados en escala Likert con cinco opciones de respuesta (Tabla 1). 
Tabla 1: Características de las facetas del módulo del WHOQOL-OLD

\begin{tabular}{lcc}
\hline Facetas & Abv. & Items en cada faceta \\
\hline "Participación" & SOP & $14+16+17+18$ \\
"Intimidad" & INT & $21+22+23+24$ \\
"Autonomía" & AUT & $3+4+5+11$ \\
"Mortalidad" & DAD & $6+7+8+9$ \\
"Habilidades Sensoriales" & SAB & $1+2+10+20$ \\
"Actividades en el tiempo" & PPF & $12+13+15+19$ \\
\hline
\end{tabular}

Nota: Elaboración propia adaptado de: WORLD HEALTH ORGANIZATION (OMS) [12, p.14]

Los datos fueron analizados y trabajados utilizando los paquetes estadísticos: SPSS 25, AMOS 20, FACTOR ANALYSIS 10.10 y R 4.

\subsection{Participantes}

El diseño muestral fue probabilístico y el tamaño de muestra considerado fue de 365 personas adultas mayores que fueron seleccionadas aleatoriamente de los que asisten a los centros de atención del adulto mayor en el distrito de Miraflores. El estudio se llevó a cabo en noviembre de 2017, mediante entrevistas personales en los centros de atención al adulto mayor.

Los resultados demográficos obtenidos fueron: $76 \%$ de los participantes fueron mujeres, un $83 \%$ de los adultos mayores es menor a 80 años de edad, $49 \%$ manifestaron haber seguido una carrera técnica o universitaria, $40 \%$ concluyó estudios secundarios, $10 \%$ culminó la primaria y un $1 \%$ son analfabetos; por otro lado, $50 \%$ indicaron ser viudos o divorciados, $39 \%$ estar casados, $11 \%$ solteros y $61 \%$ informa no tener actualmente pareja. Todos los rasgos aquí mostrados, no presentan influencia alguna en el transcurso del proceso ni sobre los resultados finales.

\section{Resultados y discusión}

Siendo que, la investigación tuvo como principal objetivo validar, determinar y cuantificar el Módulo WHOQOL-OLD de la OMS en personas atendidas en organizaciones para el adulto mayor del Distrito de Miraflores (Figura 1,) se consideró realizar el proceso en tres partes: Evaluar el modelo teórico, evaluar los constructos y generar el Modelo de segundo orden.

\section{1. $1^{\circ}$ PARTE: Evaluación del Modelo Teórico}

Se evaluó el Modelo Teórico planteado por la OMS para Calidad de Vida en adultos mayores, con finalidad de verificar si cumplía con las expectativas planteadas originalmente.

Para ello, dado que los resultados del análisis de asimetría multivariada de Mardia $(68,515)$ se encontraban en el límite de aceptación de la normalidad multivariada y siendo que todas las variables en el Modelo Teórico son de tipo ordinal ( 5 categorías), el método de estimación aplicado inicialmente fue el de máxima verosimilitud (ML), con la finalidad de facilitar la toma de decisiones sobre la validez del constructo [13].

Los resultados obtenidos en el Modelo Teórico, presentan algunos de sus principales índices de calidad de ajuste inaceptables (Tabla 2), mostrando además inapropiado el error de la varianza (casos heywood) en el factor PPF ( $66=-0,02)$. Este tipo de resultados se les conoce como estimaciones infractoras, siendo necesario la inexistencia de ellas para proceder con una 


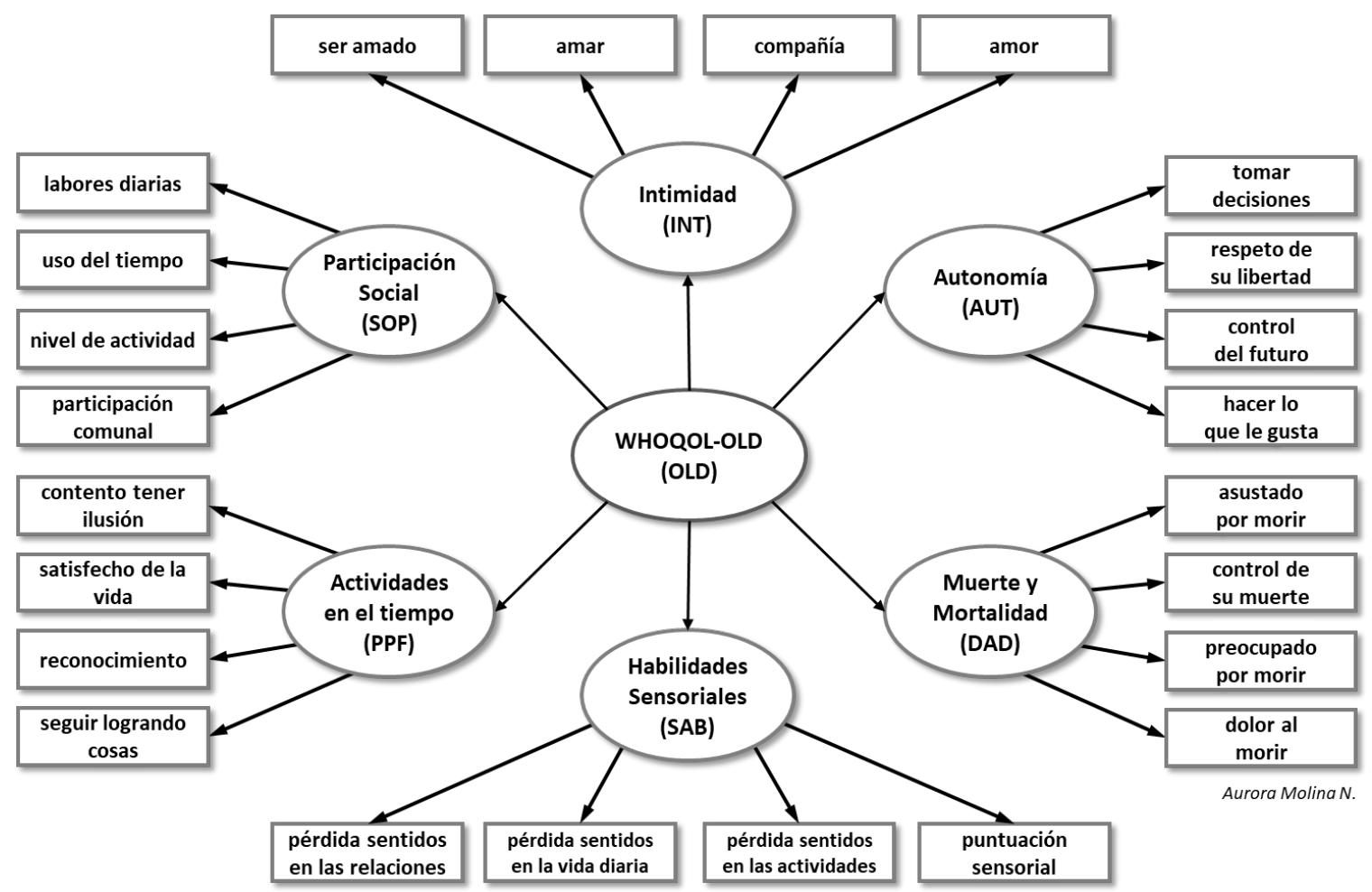

Figura 1: Modelo teórico para WHOQOL-OLD según la OMS (OLD-0)

Nota: Elaboración propia basado en el Módulo WHOQOL-OLD de la OMS

estimación correcta del modelo; además que su presencia denotará problemas en la especificación y/o identificación de este, siendo posible solucionarlo al incrementar el tamaño de muestra, modificar el modelo o fijar a un valor predeterminado los parámetros problemáticos; sea cual sea la corrección aplicada, el modelo deberá ser re-estimado [14, p. 127] [15, p. 21].

Tabla 2: Principales índices de ajuste obtenidos por máxima verosimilitud (ML) del Modelo Teórico para Calidad de Vida en adultos mayores según el Módulo WHOQOL-OLD de la OMS

\begin{tabular}{lccc}
\hline Medida & Esperado & Estimado & Interpretación \\
\hline NCS & $3>\chi 2 / g l \geqslant 2$ & 3,002 & Aceptable \\
GFI & $0,90 \leqslant G F I \leqslant 0,95$ & 0,826 & Inaceptable \\
SRMR & $0,10 \geqslant S R M R \geqslant 0,08$ & 0,119 & Inaceptable \\
RMSEA & $0,08 \geqslant R M S E A \geqslant 0,06$ & 0,079 & Aceptable \\
PClose & $0,01 \leqslant P C l o s e \leqslant 0,05$ & 0,000 & Inaceptable \\
\hline
\end{tabular}

Nota: Elaboración propia adaptado de AMOS plugin de Gaskin, J. (2016). ModelFit. Obtenido de Estadísticas de Gaskination: http://statwiki.kolobkreations.com (gl = 246)

Visto que los resultados obtenidos no satisfacen el análisis basado en la muestra, se determinó necesario verificar si el Módulo mide en realidad la Calidad de Vida en adultos mayores.

\section{2. $\quad 2^{\circ}$ PARTE: Evaluación de constructos}

\subsubsection{Análisis Factorial Exploratorio (AFE):}

Tomando en cuenta lo establecido teóricamente en WHOQOL-OLD y siendo que todas las variables son de tipo categórico, se estableció el número de factores en 6 (según lo especificado 
por OMS); utilizando para el análisis el promedio de mínimos parciales (MAP) y aplicando correlaciones policóricas (PCC) [16]; luego, con el fin de conocer cómo se agrupaban las variables, el análisis se basó en el método de extracción por mínimos cuadrados no ponderados (ULS) [17, p.1166]; por último, observando la matriz de correlación de factores con rotación oblicua, varios de sus resultados excedieron el valor $0,32(\mathrm{~F} 1 / \mathrm{F} 2=0,340 ; \mathrm{F} 1 / \mathrm{F} 4=0,464 ; \mathrm{F} 2 / \mathrm{F} 5=0,462$ y $\mathrm{F} 3 / \mathrm{F} 4=0,419)$; teniendo que existe más del $10 \%$ de probabilidad de superposición de varianzas entre factores, y por tanto, es recomendable una rotación oblicua tipo Promax [18, p.651].

Con las especificaciones mencionadas, al realizar el AFE se encontró que la varianza total explicada basada en autovalores mayores a la unidad, bajo un punto de vista numérico o matemático, advierten la necesidad de trabajar con cinco factores; reconsiderando establecer el modelo en solo cinco factores. Así el cálculo del índice de adecuación muestral Kaiser-Meyer-Olkin $(K M O=0,85)$ resultó apropiado para su identificación, poniendo en manifiesto la pertinencia de realizar el análisis factorial así planteado, siendo explicada la varianza total en un $76 \%$.

Por otro lado, en la matriz inicial de cargas rotadas, las variables (y11 y12, y21, y22 e y24) mostraron poco aporte al modelo y al ser excluidas [19] secuencialmente, después de discutir conceptualmente cada caso, se obtuvo una nueva estructura factorial (Tabla 3).

Tabla 3: Comunalidades y estructura factorial - Matriz de carga rotada para CV (ModCV-1)

\begin{tabular}{lcccccc}
\hline Variables & F1 & F2 & F3 & F4 & F5 & Comunalidades \\
\hline y01 suficientes labores diarias & 0,820 & & & & & 0,735 \\
y02 satisfacción con uso del tiempo & 0,948 & & & & & 0,910 \\
y03 satisfacción con nivel actividad & 0,901 & & & & & 0,844 \\
y04 oportunidad comunal & 0,841 & & & & & 0,683 \\
y05 oportunidad de ser amado & & 0,874 & & & & 0,775 \\
y06 oportunidad de amar & & 0,947 & & & 0,873 \\
y07 sentimiento de compañía & & 0,731 & & & & 0,577 \\
y08 sentimiento de amor & 0,905 & & & & 0,855 \\
y09 tomar decisiones & & 0,768 & & & 0,677 \\
y10 respeto de su libertad & & 0,974 & & & 0,989 \\
y13 asustado por morir & & & 0,859 & & 0,741 \\
y14 control de su muerte & & & 0,839 & & 0,707 \\
y15 preocupación de morir & & & 0,946 & & 0,876 \\
y16 dolor antes de morir & & & & 0,813 & 0,780 \\
y17 pérdida sentidos en relaciones & & & & 0,966 & 0,750 \\
y18 pérdida sentidos diario & & & & 0,969 & 0,921 \\
y19 pérdida sentidos en actividades & & & & 0,699 & 0,893 \\
y20 puntuación sensorial & & & & 0,551 \\
y23 satisfecho con lo conseguido & 0,694 & & & \\
\hline
\end{tabular}

Nota: Método de extracción: Mínimos cuadrados no ponderados (ULS)

\subsubsection{Análisis Factorial Confirmatorio (AFC):}

El AFC "constituye un caso particular de Análisis mediante Estructuras de Covarianzas que tiene como objetivo contrastar un modelo de medida con los datos obtenidos en una muestra que, teóricamente, refleja fielmente las características de la población" [14, p.119].

Luego, al correlacionar los constructos resultantes del AFE y estimarlo mediante ML, se observó la presencia de baja carga factorial estandarizada en y23 "satisfecho con lo conseguido en 
la vida" $(0,561)$, además entendiendo que y20 "puntuación sensorial" $(0,645)$, es una apreciación global de las otras tres variables del mismo constructo (F5); se consideró conveniente excluir ambas variables del modelo, obteniendo nuevos índices de ajuste satisfactorios (Tabla 4).

Tabla 4: Principales Índices de ajuste de Calidad de Vida en adultos mayores (ModCV-2)

\begin{tabular}{lccc}
\hline Medida & \multicolumn{1}{c}{ Esperado } & Estimado & Interpretación \\
\hline NCS & $3>\chi 2 / g l \geqslant 2$ & 1,451 & Excelente \\
GFI & $0,90 \leqslant G F I \leqslant 0,95$ & 0,945 & Aceptable \\
SRMR & $0,10 \geqslant S R M R \geqslant 0,08$ & 0,055 & Aceptable \\
RMSEA & $0,08 \geqslant R M S E A \geqslant 0,06$ & 0,036 & Excelente \\
PClose & $0,01 \leqslant P C l o s e \leqslant 0,05$ & 0,956 & Excelente \\
\hline
\end{tabular}

Nota: Elaboración propia adaptado de AMOS plugin de Gaskin, J. (2016). ModelFit por ML.

Obtenido de Estadísticas de Gaskination: http://statwiki.kolobkreations.com ( $\mathrm{gl}=109)$

Luego, la estimación de las covarianzas entre factores, Figura 2 (Modelo de Medida)

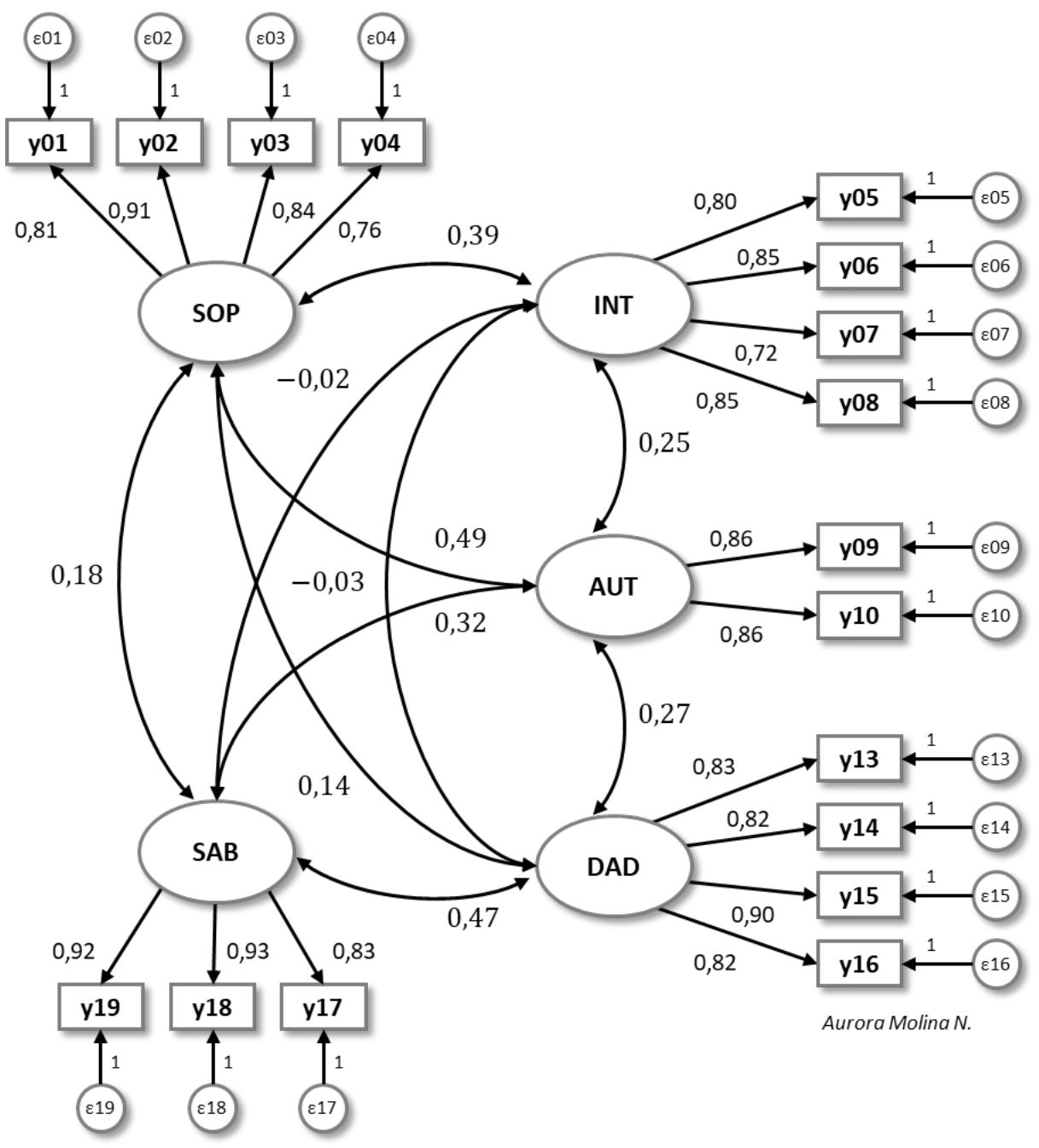

Figura 2: Valores estandarizados estimador por máxima verosimilitud del Modelo de medida para el módulo WHOQOL-OLD (Mod-1)

Nota: Elaboración propia 
se observa: INT/DAD $(-0,026)$ e INT/SAB $(-0,016)$ no son estadísticamente significativas, con p-valor de 0,673 y 0,790 respectivamente, y al no existir evidencia que relacione estos factores y con la finalidad de obtener una mejor parsimonia, sería posible excluirlas del modelo (Modelo de Medida Incompleto); sin embargo, para fines de nuestro estudio su presencia no causa mayor dificultad, pudiendo sin problema continuar con el proceso.

La Figura 2 muestra los valores estandarizados obtenidos mediante máxima verosimilitud en el Modelo de Medida para el Módulo WHOQOL-OLD de la OMS, donde se aprecian cinco variables latentes representadas mediante círculos $(\xi)$, con un total de diecisiete variables observables (y) representadas por rectángulos con sus respectivas perturbaciones en círculos más pequeños $(\epsilon)$, las saturaciones o coeficientes de regresión son determinados por flechas unidireccionales $(\lambda)$ que van del factor a sus respectivas variables observables con la que están relacionadas, estableciendo en la unidad uno de sus indicadores como referencia; además de que se muestran las covarianzas entre factores $(\phi)$.

Expresando dichas relaciones mediante las siguientes ecuaciones:

$$
\begin{array}{lll}
y_{01}=\xi_{1}+\epsilon_{01} & y_{07}=\lambda_{072} \xi_{2}+\epsilon_{07} & y_{15}=\lambda_{15} \xi_{4}+\epsilon_{15} \\
y_{02}=\lambda_{02} \xi_{1}+\epsilon_{02} & y_{08}=\lambda_{08} \xi_{2}+\epsilon_{08} & y_{16}=\lambda_{16} \xi_{4}+\epsilon_{16} \\
y_{03}=\lambda_{03}{ }_{1} \xi_{1}+\epsilon_{03} & y_{09}=\xi_{3}+\epsilon_{09} & y_{17}=\xi_{5}+\epsilon_{17} \\
y_{04}=\lambda_{04} \xi_{1}+\epsilon_{04} & y_{10}=\lambda_{103} \xi_{3}+\epsilon_{10} & y_{18}=\lambda_{18} \xi_{5}+\epsilon_{18} \\
y_{05}=\xi_{2}+\epsilon_{05} & y_{13}=\xi_{4}+\epsilon_{13} & y_{19}=\lambda_{18} \xi_{5}+\epsilon_{19} \\
y_{06}=\lambda_{06} \xi_{2}+\epsilon_{06} & y_{14}=\lambda_{14} \xi_{4}+\epsilon_{14} &
\end{array}
$$

O en su forma matricial:

$$
\left[\begin{array}{l}
y_{01} \\
y_{02} \\
y_{03} \\
y_{04} \\
y_{05} \\
y_{06} \\
y_{07} \\
y_{08} \\
y_{09} \\
y_{10} \\
y_{13} \\
y_{14} \\
y_{15} \\
y_{16} \\
y_{17} \\
y_{18} \\
y_{19}
\end{array}\right]=\left[\begin{array}{ccccc}
1 & 0 & 0 & 0 & 0 \\
\lambda y_{02} 1 & 0 & 0 & 0 & 0 \\
\lambda y_{03} 1 & 0 & 0 & 0 & 0 \\
\lambda y_{04} 1 & 0 & 0 & 0 & 0 \\
0 & 1 & 0 & 0 & 0 \\
0 & \lambda y_{06} 2 & 0 & 0 & 0 \\
0 & \lambda y_{072} & 0 & 0 & 0 \\
0 & \lambda y_{08} 2 & 0 & 0 & 0 \\
0 & 0 & 1 & 0 & 0 \\
0 & 0 & \lambda y_{103} & 0 & 0 \\
0 & 0 & 0 & 1 & 0 \\
0 & 0 & 0 & \lambda y_{144} & 0 \\
0 & 0 & 0 & \lambda y_{15} 4 & 0 \\
0 & 0 & 0 & \lambda y_{164} & 0 \\
0 & 0 & 0 & 0 & 1 \\
0 & 0 & 0 & 0 & \lambda y_{185} \\
0 & 0 & 0 & 0 & \lambda y_{19} 5
\end{array}\right]\left[\begin{array}{c}
\xi_{1} \\
\xi_{2} \\
\xi_{3} \\
\xi_{4} \\
\xi_{5}
\end{array}\right]+\left[\begin{array}{c}
\epsilon_{01} \\
\epsilon_{02} \\
\epsilon_{03} \\
\epsilon_{04} \\
\epsilon_{05} \\
\epsilon_{06} \\
\epsilon_{07} \\
\epsilon_{08} \\
\epsilon_{09} \\
\epsilon_{10} \\
\epsilon_{13} \\
\epsilon_{14} \\
\epsilon_{15} \\
\epsilon_{16} \\
\epsilon_{17} \\
\epsilon_{18} \\
\epsilon_{19}
\end{array}\right]
$$

Con covarianzas:

$$
\begin{array}{lll}
\operatorname{Cov}\left(\xi_{1}, \xi_{2}\right)=\phi_{21} & \operatorname{Cov}\left(\xi_{1}, \xi_{3}\right)=\phi_{31} & \operatorname{Cov}\left(\xi_{1}, \xi_{4}\right)=\phi_{41} \\
\operatorname{Cov}\left(\xi_{1}, \xi_{5}\right)=\phi_{51} & \operatorname{Cov}\left(\xi_{2}, \xi_{3}\right)=\phi_{32} & \operatorname{Cov}\left(\xi_{2}, \xi_{4}\right)=\phi_{42} \\
\operatorname{Cov}\left(\xi_{2}, \xi_{5}\right)=\phi_{52} & \operatorname{Cov}\left(\xi_{3}, \xi_{4}\right)=\phi_{43} & \operatorname{Cov}\left(\xi_{3}, \xi_{5}\right)=\phi_{53} \\
\operatorname{Cov}\left(\xi_{4}, \xi_{5}\right)=\phi_{54} & &
\end{array}
$$

Luego de especificar el modelo, se procedió a examinar la valoración de la fiabilidad individual de los ítems, observando que todos sus indicadores poseen cargas superiores a 0,7, asegurando 
así que la varianza compartida entre constructo e indicadores es mayor que la varianza del error [20, p.509], verificando luego:

La valoración de la confiabilidad del constructo mediante la confiabilidad compuesta:

$$
\begin{aligned}
C R_{S O P}= & 0,899 \quad C R_{I N T}=0,882 \quad C R_{A U T}=0,850 \\
& C R_{D A D}=0,907 \quad C R_{S A B}=0,920
\end{aligned}
$$

Varianza media extraída superior a 0,5 :

$$
\begin{gathered}
A V E_{S O P}=0,691 \quad A V E_{I N T}=0,651 \quad A V E_{A U T}=0,739 \\
A V E_{D A D}=0,709 \quad A V E_{S A B}=0,794
\end{gathered}
$$

Y la validez discriminante comparando la varianza media extraída de cada constructo, con el cuadrado de sus correlaciones; cumpliendo con ser mayores que entre los demás factores:

$$
\begin{gathered}
\sqrt{A V E_{S O P}}=0,831>\left\{\begin{array}{c}
0,394=\operatorname{cor}(S O P, I N T) \\
0,490=\operatorname{cor}(S O P, A U T) \\
0,142=\operatorname{cor}(S O P, D A D) \\
0,182=\operatorname{cor}(S O P, S A B)
\end{array}\right\} \\
\sqrt{A V E_{I N T}}=0,807>\left\{\begin{array}{c}
0,394=\operatorname{cor}(I N T, S O P) \\
0,254=\operatorname{cor}(I N T, A U T) \\
-0,026=\operatorname{cor}(I N T, D A D) \\
-0,016=\operatorname{cor}(I N T, S A B)
\end{array}\right\} \\
\sqrt{A V E_{A U T}}=0,860>\left\{\begin{array}{c}
0,490=\operatorname{cor}(A U T, S O P) \\
0,254=\operatorname{cor}(A U T, I N T) \\
0,271=\operatorname{cor}(A U T, D A D) \\
0,324=\operatorname{cor}(A U T, S A B)
\end{array}\right\} \\
\sqrt{A V E_{D A D}}=0,842>\left\{\begin{array}{c}
0,142=\operatorname{cor}(D A D, S O P) \\
-0,026=\operatorname{cor}(D A D, I N T) \\
0,271=\operatorname{cor}(D A D, A U T) \\
0,467=\operatorname{cor}(D A D, S A B)
\end{array}\right\} \\
\sqrt{A V E_{S A B}}=0,891>\left\{\begin{array}{c}
0,182=\operatorname{cor}(S A B, S O P) \\
-0,016=\operatorname{cor}(S A B, I N T) \\
0,324=\operatorname{cor}(S A B, A U T) \\
0,467=\operatorname{cor}(S A B, D A D)
\end{array}\right\}
\end{gathered}
$$

Se afirmar que los ítems correspondientes a un factor, no aportan o explican otro y viceversa.

\section{3. $\quad 3^{\circ}$ PARTE: Generación de Modelo de Segundo Orden}

En la generación del Modelo de segundo orden u orden superior, se establece la creación de un nuevo factor $(\mathrm{OLD})\left(\xi_{0}\right)$, donde las flechas que salen de él $(\gamma)$ muestran la influencia directa sobre cada una de las Variables latentes de primer nivel (SOP, INT, AUT, DAD y SAB), que ahora son consideradas como si fueran Variables observables $(\eta)$ y por tanto presentan errores estructurales $(\zeta)$ [21] (Figura 3).

Luego de establecido el Modelo estructural de segundo orden para OLD, en la determinación de sus grados de libertad, donde las variables latentes consideradas, son solamente las correspondientes al Modelo de orden superior $(\mathrm{p}=5)$ y el número de parámetros libres a ser estimados está dado por $\mathrm{t}=(1 \xi, 4 \gamma, 5 \zeta)=10$; se tiene:

$$
g l=1 / 2[p(p+1)]-t=15-10=5
$$




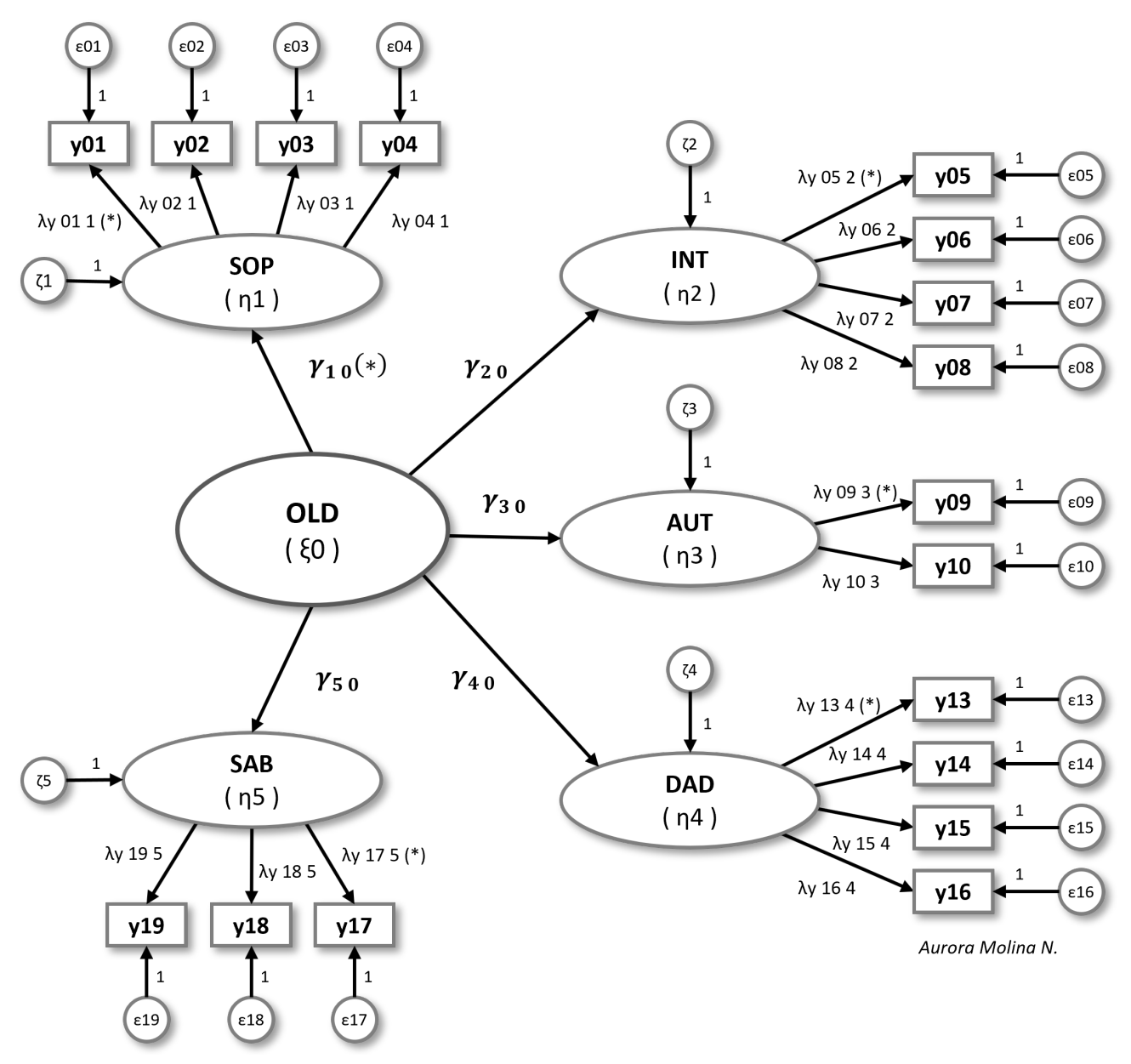

Figura 3: Constructo 2do orden para el Módulo WHOQOL-OLD de la OMS (Mod-2)

Nota: Elaboración propia $(*)$ indicadores establecidos en la unidad $(\mathrm{gl} .=114)$

Ahora bien, habiendo comprobado todos los requerimientos, el modelo estructural de 2do orden así planteado para el Módulo WHOQOL-OLD de la OMS sobre la Calidad de Vida de adultos mayores, se encuentra apto para continuar con el análisis estructural, donde sus ecuaciones referidas a las variables exógenas están dadas por:

$$
\begin{gathered}
\eta_{1}=\gamma_{10} \xi_{0}+\zeta_{1} \quad \eta_{2}=\gamma_{20} \xi_{0}+\zeta_{2} \quad \eta_{3}=\gamma_{30} \xi_{0}+\zeta_{3} \\
\eta_{4}=\gamma_{40} \xi_{0}+\zeta_{4} \quad \eta_{5}=\gamma_{50} \xi_{0}+\zeta_{5}
\end{gathered}
$$

Hasta aquí, las estimaciones han sido realizadas mediante máxima verosimilitud (ML) con el fin de facilitar su análisis; sin embargo, "cuando se trata de variables ordinales con tres o más categorías, se utilizará una matriz de correlaciones policóricas" [13, p.20], no es válido realizar estimaciones por ML, siendo necesario estimarlo mediante otras técnicas apropiadas como la estimación por Mínimos cuadrados de libre escala (SLS) [22] [23].

Estimados los parámetros mediante SLS, se evaluó el Modelo estructural, no observando la existencia de valores no significativos estadísticamente, desviaciones estándar muy altas, ni coeficientes estandarizados mayores a la unidad (Figura 4).

Además las diferencias entre los principales índices de bondad de ajuste, tanto bajo el método de ML como SLS, es mínima, mostrándose entre aceptables y excelentes (Tabla 5). 


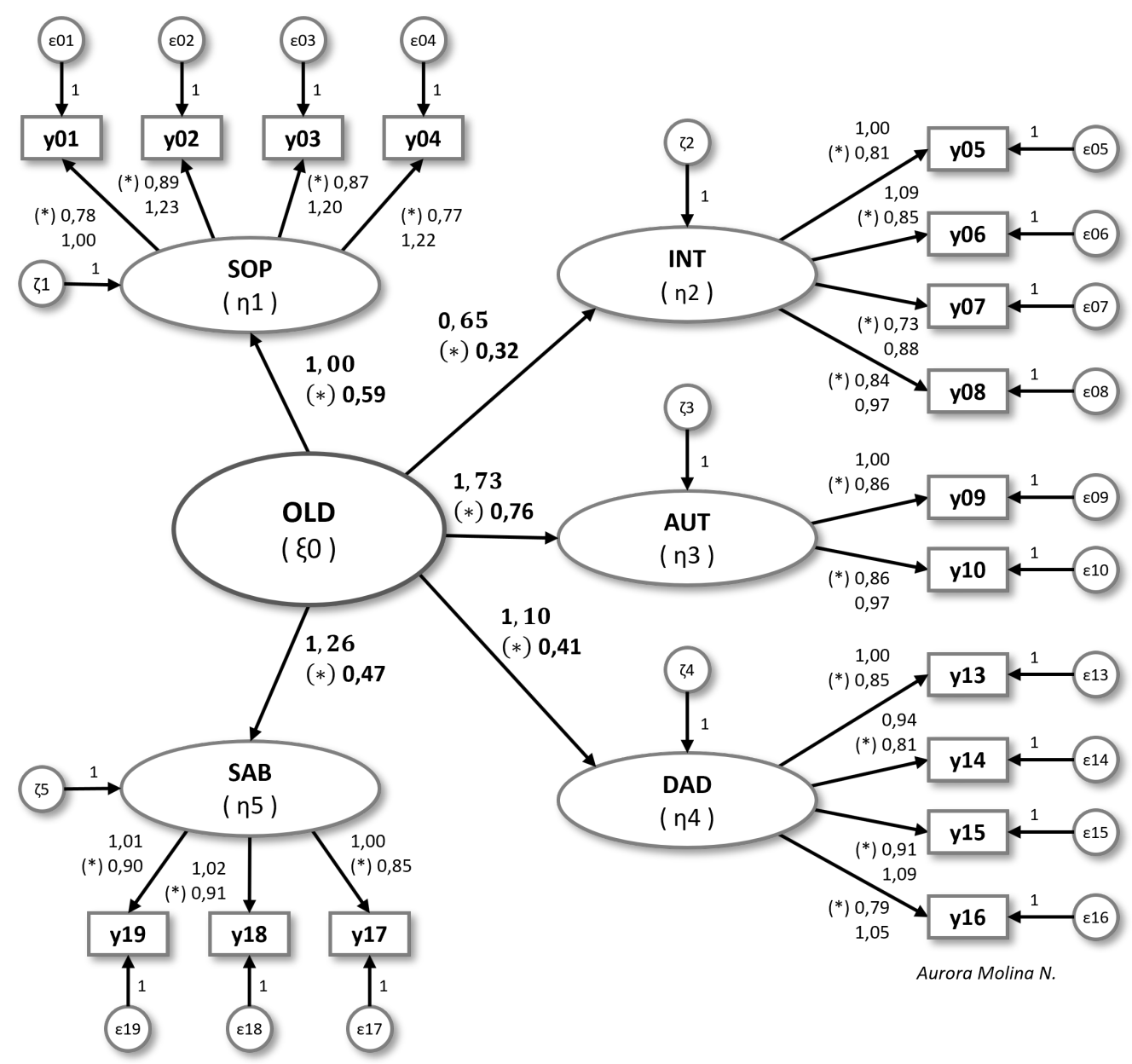

Figura 4: Estimación por mínimos cuadrados de libre escala (SLS) del Modelo de 2do orden para el Módulo WHOQOL-OLD de la OMS (Mod-3)

Nota: Elaboración propia $(*)$ valores estandarizados $(\mathrm{gl} .=114)$

Tabla 5: Principales Índices de ajuste por mínimos cuadrados de libre escala - Modelo Final

\begin{tabular}{lccccc}
\hline \multirow{2}{*}{ Medida } & \multirow{2}{*}{ Esperado } & Estimado & Interpretación & Estimado & Interpretación \\
\hline GFI & $0,90 \leqslant G F I \leqslant 0,95$ & 0,92 & Aceptable & 0,94 & Aceptable \\
SRMR & $0,10 \geqslant S R M R \geqslant 0,08$ & 0,10 & Aceptable & 0,10 & Aceptable \\
CFI & $0,90 \leqslant C F I \leqslant 0,95$ & 0,97 & Exelente & 0,94 & Aceptable \\
NFI & $0,90<N F I \leqslant 0,95$ & 0,94 & Aceptable & 0,90 & Aceptable \\
PGFI & $0,50 \leqslant P G F I \leqslant 0,70$ & 0,69 & Aceptable & 0,70 & Aceptable \\
\hline
\end{tabular}

Nota: Elaboración propia. $(\mathrm{gl}=340)$

Luego, se verifica que los coeficientes de determinación $\left(\mathrm{R}^{2}\right)$ (Tabla 6), pese que no es posible llevar a cabo algún test de significancia estadística, brindan una medida de ajuste relativa en cada constructo del modelo. 
Tabla 6: Principales coeficientes de determinación $\left(\mathrm{R}^{2}\right)$ del Modelo de 2do orden para el Módulo WHOQOL-OLD de la OMS para Calidad de Vida en adultos mayores

\begin{tabular}{lcc}
\hline Parámetro & ML & SLS \\
\hline SOP & 0,397 & 0,352 \\
INT & 0,126 & 0,100 \\
AUT & 0,589 & 0,582 \\
DAD & 0,133 & 0,165 \\
SAB & 0,170 & 0,223 \\
\hline
\end{tabular}

Nota: Elaboración propia

Adicionalmente para evaluar la fuerza y dirección de la relación entre variables, se presenta tanto la matriz de correlaciones policóricas con sus principales tendencias y el alfa ordinal policórico [24] para cada item (Tabla 7); como su correspondiente matriz visual (Figura 5), donde las correlaciones más altas se encuentran, efectivamente en cada constructo.

Tabla 7: Matriz de correlaciones policóricas para el Modelo Final de Calidad de Vida en adultos mayores basado en el Módulo WHOQOL-OLD de la OMS

\begin{tabular}{|c|c|c|c|c|c|c|c|c|c|c|c|c|c|c|c|c|c|}
\hline variables & y01 & y02 & y03 & y04 & y05 & y06 & y07 & y08 & y09 & y10 & y13 & y14 & $\mathrm{y} 15$ & $\mathrm{y} 16$ & y17 & y18 & y19 \\
\hline y01 & 1 & & & & & & & & & & & & & & & & \\
\hline y02 & 0,84 & 1 & & & & & & & & & & & & & & & \\
\hline y03 & 0,75 & 0,87 & 1 & & & & & & & & & & & & & & \\
\hline y04 & 0,67 & 0,76 & 0,74 & 1 & & & & & & & & & & & & & \\
\hline y05 & 0,35 & 0,34 & 0,27 & 0,21 & 1 & & & & & & & & & & & & \\
\hline y06 & 0,37 & 0,34 & 0,27 & 0,28 & 0,69 & 1 & & & & & & & & & & & \\
\hline y07 & 0,37 & 0,33 & 0,27 & 0,14 & 0,67 & 0,67 & 1 & & & & & & & & & & \\
\hline y08 & 0,37 & 0,39 & 0,33 & 0,30 & 0,70 & 0,78 & 0,64 & 1 & & & & & & & & & \\
\hline y09 & 0,35 & 0,37 & 0,41 & 0,35 & 0,21 & 0,18 & 0,15 & 0,11 & 1 & & & & & & & & \\
\hline y10 & 0,38 & 0,42 & 0,44 & 0,38 & 0,28 & 0,26 & 0,25 & 0,18 & 0,80 & 1 & & & & & & & \\
\hline y13 & 0,16 & 0,16 & 0,23 & 0,16 & 0,02 & 0,10 & $-0,03$ & 0,00 & 0,25 & 0,20 & 1 & & & & & & \\
\hline y14 & 0,07 & 0,09 & 0,13 & 0,11 & $-0,01$ & $-0,01$ & $-0,02$ & $-0,11$ & 0,22 & 0,22 & 0,71 & 1 & & & & & \\
\hline y15 & 0,12 & 0,12 & 0,15 & 0,20 & $-0,02$ & 0,02 & $-0,04$ & $-0,09$ & 0,25 & 0,22 & 0,80 & 0,77 & 1 & & & & \\
\hline y16 & 0,02 & 0,09 & 0,14 & 0,13 & $-0,11$ & $-0,06$ & $-0,17$ & $-0,15$ & 0,17 & 0,11 & 0,74 & 0,71 & 0,79 & 1 & & & \\
\hline y17 & 0,06 & 0,15 & 0,17 & 0,13 & $-0,05$ & $-0,03$ & $-0,05$ & 0,05 & 0,28 & 0,24 & 0,38 & 0,41 & 0,46 & 0,48 & 1 & & \\
\hline y18 & 0,06 & 0,17 & 0,22 & 0,09 & $-0,05$ & $-0,08$ & $-0,04$ & $-0,03$ & 0,32 & 0,27 & 0,36 & 0,36 & 0,41 & 0,46 & 0,80 & 1 & \\
\hline y19 & 0,08 & 0,18 & 0,24 & 0,13 & $-0,02$ & $-0,04$ & $-0,05$ & 0,00 & 0,27 & 0,22 & 0,37 & 0,34 & 0,38 & 0,45 & 0,80 & 0,90 & 1 \\
\hline $\mathrm{M}$ & 3,49 & 3,50 & 3,34 & 3,50 & 3,55 & 3,43 & 3,40 & 3,55 & 3,65 & 3,70 & 3,18 & 3,17 & 3,23 & 2,78 & 3,29 & 3,09 & 3,01 \\
\hline $\mathrm{DE}$ & 0,81 & 0,88 & 0,87 & 0,93 & 0,96 & 1,00 & 0,95 & 0,91 & 1,00 & 0,97 & 1,20 & 1,19 & 1,23 & 1,34 & 1,18 & 1,13 & 1,13 \\
\hline Fiabilidad & 0,85 & 0,85 & 0,85 & 0,85 & 0,86 & 0,86 & 0,86 & 0,86 & 0,86 & 0,85 & 0,86 & 0,86 & 0,86 & 0,86 & 0,86 & 0,86 & 0,86 \\
\hline
\end{tabular}

Nota: Elaboración propia. 


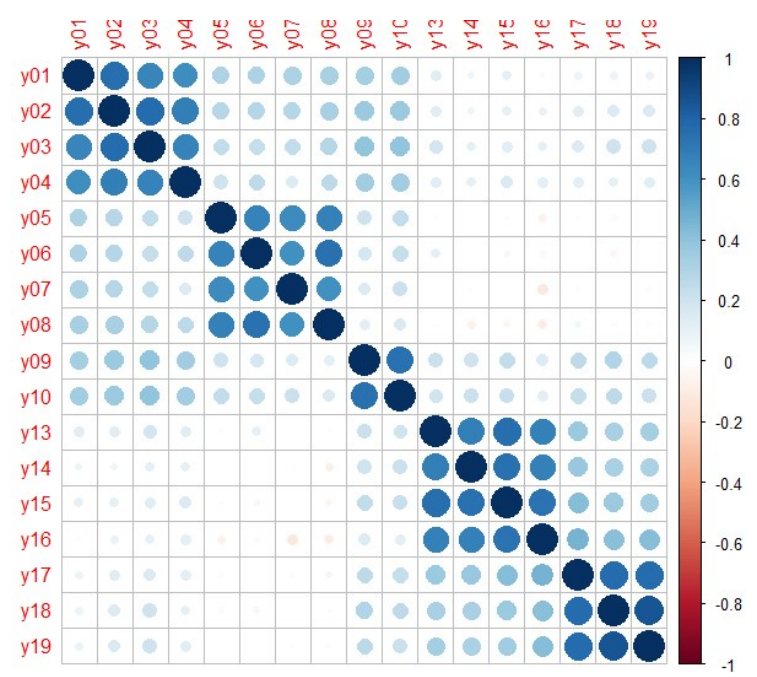

Figura 5: Matriz de correlaciones policóricas para el Modelo Final de Calidad de Vida en adultos mayores, basado en el Módulo WHOQOL-OLD de la OMS

Nota: Elaboración propia. Tamaño y saturación de color en función de la fuerza correlacional

\section{Conclusión}

En conclusión, el Módulo WHOQOL-OLD de la OMS ha permitido evaluar la Calidad de Vida de las personas atendidas en organizaciones para el adulto mayor institucionalizados (distrito de Miraflores). Los resultados obtenidos, confirman parcialmente las hipótesis de investigación, siendo que una de sus seis dimensiones (actividades pasadas, presentes y futuras) no fue posible validar, obteniendo un nuevo modelo con evidencia suficiente de ajuste, validez y fiabilidad que prueban la relación entre sus constructos, sugiriendo una posterior revisión en muestras afines.

Los principales hallazgos encontrados en el nuevo Modelo se refieren a:

- Las altas valoraciones de la Autonomía, principalmente sobre el hecho del respeto por su libertad en la cuantificación de la Calidad de Vida en adultos mayores.

- El alto nivel de explicación dado por las variables independientes de Calidad de Vida de adultos mayores; específicamente al hecho referido a la pérdida de los sentidos en la vida diaria, en su intención de cuantificar las Habilidades Sensoriales.

- El bajo reflejo de la Calidad de Vida sobre la Intimidad, específicamente al hecho de la menor valoración sobre los sentimientos de compañía.

- La poca importancia o no relevancia de las Actividades pasadas, presentes y futuras referidas a la Calidad de Vida en adultos mayores.

La presente investigación muestra cómo la aplicación de los Modelos de Ecuaciones Estructurales no solo obtiene resultados acordes a la realidad; sino supone también una herramienta de amplia utilidad para análisis de investigaciones futuras, pudiendo incluir en él, variables latentes (que evalúen relaciones y efectos), sometiéndolas a una comprobación simultánea en la estructura completa, obteniendo pruebas que se basen en la congruencia del modelo hipotetizado con nuevas realidades. Sin embargo, sería interesante validarlo en nuevos estudios con muestras equivalentes y, muy posiblemente, utilizarlo como explicación para teorías de mayor complejidad que se deseen contrastar, como el hecho de la situación actual en el mundo sobre la presencia de la pandemia por el COVID-19. 


\section{Referencias bibliográficas}

[1] OMS. (2020). Temas de salud: Envejecimiento. Recuperado de https://www.who.int/ topics/ageing/es/

[2] INEI. (2020). Situación de la Población Adulta Mayor. Lima. https://www.inei.gob.pe/ media/MenuRecursivo/boletines/informe-tecnico-poblacion-adulta-mayor .pdf

[3] Molina, A.-del C.(2021). Relación entre el nivel de dependencia de funcionalidad de las actividades cotidianas y la calidad de vida de personas atendidas en organizaciones para el adulto mayor. (TESIS entregado para la publicación). Universidad Nacional Mayor de San Marcos, Lima

[4] Rodríguez-Chacaltana, F. W., Quispe-Ilanzo, M. P., Oyola-García, A. E., De La Cruz, C. Y. C., Portugal-Medrano, M. A., Lizarzaburu-Córdova, E. E. y Mejía-Vargas, E. (2018). Calidad de vida y su relación con la malnutrición en el adulto mayor de la provincia de Ica. Revista de la Facultad de Medicina Humana, 18(4), 1-1

[5] Aguilar Navarro, M. R., Alonso Rivera, C. E., González Castro, S., Juárez Nieto, M. A., Ponce Moreno, V. y Landeros-López, M. CALIDAD DE VIDA EN EL ADULTO MAYOR CON ENFERMEDADES CRÓNICAS EN EL GRUPO "UN DÍA MÁS"

[6] Zapata Mamani, K. Y. y Millones Saldaña, V. M. (2019). LA ACTIVIDAD FÍSICA COMO INTERVENCIÓN PARA LA MEJORA DE LA CALIDAD DE VIDA EN EL ADULTO MAYOR

[7] PNDH. (2017). Plan Nacional de Derechos Humanos 2018-2021. Lima: Ministerio de Justicia y Derechos Humanos (MINJUSDH)

[8] Queirolo Ore, S. A., Barboza Palomino, M. y Ventura-León, J. (2020). Medición de la calidad de vida en adultos mayores institucionalizados de Lima (Perú). Enfermería Global, $19(60), 259-288$.

[9] Merino Diaz, E. M., Olano Rodriguez, S. y Paucar Espinoza, J. L. (2017). Nivel de dependencia y la percepción de la calidad de vida de los adultos mayores del Centro de Atención Residencial Geriátrico San Vicente de Paúl La Victoria 2016.

[10] Leguia Cerron, A. D. P. Calidad de vida y estrategias de afrontamiento en adultos mayores de Lima Metropolitana.

[11] Power, M., Quinn, K. y Schmidt, S. (2006). Development of the WHOQOL-OLD module. Quality of Life Research, 2197-2214. doi:10.1007/s11136-005-7380-9

[12] OMS. (2006). Manual WHOQOl-OLD. (E. O. COPENHAGEN Ed.) Recuperado de WORLD HEALTH ORGANIZATION: https://www.who.int/mental_health/ evidence/WHOQOL_OLD_Manual.pdf?ua=1Manual WHOQOL-OLD de la OMS

[13] Mangin, J., Fuentes, M. y González, M. (2006a). Optimización según estructuras de covarianzas. En J. Mangin y J. Mallou, Modelización con Estructuras de Covarianzas en Ciencias Sociales: Temas esenciales, avanzados y aportaciones especiales (págs. 11-30). Madrid: Netbiblo

[14] Baubeta, A., Mallou, J., Piñeiro, J. y Mangin, J. (2006). El Análisis factorial confirmatorio. En J. Mangin y J. Mallou, Modelización con Estructuras de Covarianzas en Ciencias Sociales: temas esenciales, avanzados y aportaciones especiales (págs. 119-154). Madrid: Netbiblo 
[15] Alonso, M., Álvarez, N. y Doyague, M. (2006). Introducción a los Modelos de Estructuras de Covarianzas. En J. Mangin y J. Mallou, Modelización con Estructuras de Covarianzas en Ciencias Sociales: temas esenciales, avanzados y aportaciones especiales (págs. 1-10). Madrid: Netbiblo

[16] Velicer, W. F. (1976). Determining the number of components from the matrix of partial correlations. Psychometrika, 41(3), 321-327. doi:10.1007/BF02293557

[17] Lloret, S., Ferreres, A., Hernández, A. y Tomás, I. (2014). El análisis factorial exploratorio de los ítems: una guía práctica, revisada y actualizada. Anales de Psicología, 30 (3), 11511169. doi:10.6018/analesps.30.3.199361

[18] Tabachnick, B. y Fidell, L. (2001). Using Multivariate Statistics (4th Edition ed.). Allyn \& Bacon: Allyn and Bacon. doi:ISBN 0-321-05677-9

[19] Hair Jr., J., Anderson, R., Tatham, R. y Black, W. (1999). Análisis multivariante. Madrid: Prentice Hall

[20] Chin W. y Dibbern J. (2006). A Permutation Based Procedure for multi-Group Pls Analysis: results of tests of differences on simulated data and a cross-cultural analysis of the sourcing of information system services Between Germany And the USA. En J. Mangin y J. Mallou, Modelización con Estructuras de Covarianzas en Ciencias Sociales: temas esenciales, avanzados y aportaciones especiales (págs. 501-517). Madrid: Netbiblo

[21] Marín García, Juan Antonio (2014). EQS-validación de constructos de segundo orden. http: //hdl.handle.net/10251/38736

[22] Kaplan, D. (2000). Structural Equation Modeling: Foundations and Extensions (2da edición ed.). Thousand Oaks, CA: Sage Publications

[23] Browne, A. (1982). Covariance structures. En D. Hawkins, Topics in applied multivariate analysis (págs. 72-141). Cambridge: Cambridge University Press

[24] Zumbo, B. D., Gadermann, A. M. y Zeisser, C. (2007). Ordinal versions of coefficients alpha and theta for Likert rating scales. Journal of modern applied statistical methods, 6(1), 4 